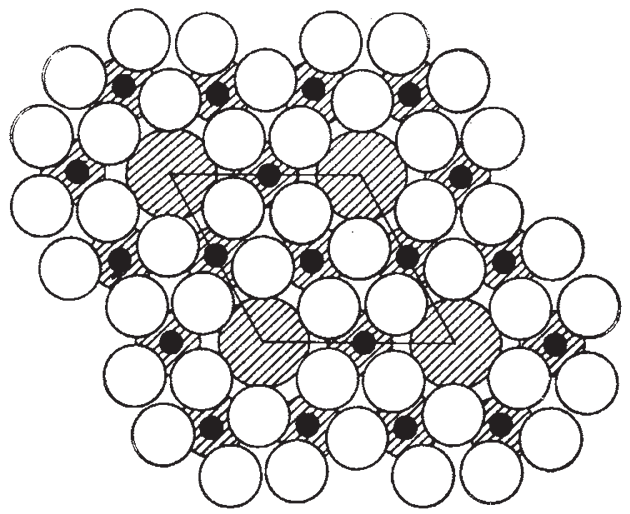

Filled small circle, tungsten; medium circle, oxygen; large circle, alkali metal

of the lattice, thus co-ordinating twelve oxygen atoms. The theoretical upper limit of the alkali content evidently corresponds to a value of $x$ of $1 / 3$ in the formula given above.

The structure offers another example of the broad extent of the possible combinations of the $\mathrm{WO}_{6}$ octahedra in tungsten-oxygen compounds, manifested in various tungsten oxides ${ }^{2}$, tungsten bronzes ${ }^{2}$, and heteropolytungstates ${ }^{3}$. Six-membered rings of $\mathrm{WO}_{6}$ octahedra have previously been encountered only in the tungsten oxide $\mathrm{W}_{18} \mathrm{O}_{49}$, of complicated structure, where, however, they are arranged around empty channels of infinite extension running through the lattice ${ }^{4}$.

Full details of this investigation will shortly appear elsewhere.

Institute of Chemistry,

ARne Magnéli

University of Uppsala. Dec. 15.

1 Magnéli, A., and Blomberg, B., Acta Chem. Scand., 5, 372 (1951). 'Magnéli, A., Nova Acta Regiae Soc. Sci. Upsaliensis, [4], 14, No. \& (1950).

"Wells, A. F., "Structural Inorganic Chemistry" (2nd edit., Oxford,

4 Magnéli, A., Arkiv Kemi, 1, 223 (1949).

\section{Removal of Impurities from Tin by Vacuum Distillation}

TrE low vapour pressure of tin at temperatures up to $1,000^{\circ} \mathrm{C}$. makes it possible to remove more volatile constituents of tin-rich alloys by vacuum distillation. The use of this method has been described for the analytical determination of cadmium, tellurium, zinc, lead and phosphorus ${ }^{1}$.

I have since used this procedure in attempts to remove the last traces of certain impurities from samples of refined tin: A specimen of tin was heated in an evacuated silica tube at $1,000^{\circ} \mathrm{C}$. for four hours, after which a spectrographic examination showed arsenic, antimony, lead and bismuth to be absent. As there is some uncertainty as to the limits of spectrographic detection of these elements, a colorimetric method was used for the determination of arsenic, and this showed the arsenic content to be less than 0.0002 per cent. From this it seems that vacuum distillation is a promising method for the production of tin of very high purity.

In all the above work a pressure of about $0.05 \mathrm{~mm}$. mercury was maintained during distillation by means of a rotary oil pump, and the evolved metal condensed in a ring on the cooler part of the silica tube. I have recently included a diffusion pump in the pumping circuit giving a pressure of about $10^{-5} \mathrm{~mm}$. during distillation, and I have found that some separation of the evolved metal vapours can be obtained. In one experiment, three distinct bands condensed on the wall of the tube. These consisted of an outer black band mainly of antimony and an inner grey band mainly of tin, separated by a thin brown band, which has not yet been identified. This suggests a method for the separation and possible determination of trace impurities in tin. Work is continuing along these lines and will be reported elsewhere.

Tin Research Institute,

J. W. Price

Fraser Road,

Perivale,

Greenford, Middlesex. Dec. 19.

I Price, J. W., J. Soc. Chem. Indust., 44, 283 (1945).

\section{Galvanic Determination of Traces of Oxygen in Gases}

THE cathodic reduction of oxygen has been studied ${ }^{2}$ in connexion with corrosion and air-depolarized galvanic cells; but so far no use seems to have been made of this process in gas analysis. $X$ have experimented with cathodes half-immersed in the electrolyte under nitrogen and hydrogen and, with suitably designed cells, have found that otherwise difficultly detectable traces of oxygen in the gas phase, such as $10^{-3}$ c.c./c.c., were capable of giving substantial and easily measurable currents.

The diagram shows one of several types of cell tried as a 'reversed gas coulomb-meter'. The vessel is flushed by several evacuation-admission cycles. On closing the circuit over $1,000 \mathrm{ohms}$, there is at first a surge of current, owing to oxygen adsorbed on the platinum cathode. After this, if some 0.01 per cent oxygen is present, the current is limited by the depolarizing power of the cathode water-line. When about 0.001 per cent oxygen is present, or left, oxygen diffusion in the gas space takes over control and the current declines logarithmically.

To avoid plotting a curve and integrating, the current can be damped down to a chosen constant value, for example, $20 \mu a m p$., by using a high e.m.f. plus a high resistance in series with the cell. What is then relevant is the time required for the terminal voltage of the cell to be reversed in sign. This moment, is sharply defined, though early warning is given of its approach. The auxiliary part of the circuit is then shorted out by closing the switch $S$, so that the current is allowed to decline. Otherwise plumbite and later water would be reduced along with oxygen. No further observations are, however, nécessary if the 'tail', $\int_{i=20}^{i=0} i \mathrm{~d} t$, that is, the residual oxygen, has been determined in one previous run, as an apparatus constant.

On introducing measured traces of air, the number of coulombs obtained on discharge has been compared with that calculated from Faraday's law, assuming reduction of oxygen to hydroxyl. The measured results are some 5 per cent lower than the calculated figure, possibly owing to some oxygen being reduced only to hydroperoxyl. With the cell described, oxygen 